\title{
Seismic Imaging of Scatterer Migration Associated with the 2004 Parkfield Earthquake Using Waveform Data of Repeating Earthquakes and Active Sources
}

\author{
by Xin Cheng, Fenglin Niu, Paul G. Silver, and Robert M. Nadeau
}

\begin{abstract}
We used borehole seismic records of four repeating-earthquake clusters and two explosions to investigate the coseismic changes of the scattering wave field of the 28 September $2004 M 6$ Parkfield earthquake. We found systematic changes in $P$-wave and $S$-wave coda recorded from repeating events that occurred before and immediately after the earthquake. Applying the coda wave interferometry technique allowed us to determine that the observed changes are caused by a localized change in the scattering field. We further developed a technique based on decorrelation indexes calculated from running time windows to locate migrating scatterers. Synthetic tests showed that the technique is relatively insensitive to changes in background velocity of the medium and source location and thus can be applied to records of loosely colocated clusters. We found a localized change of material property within the fault zone at $\sim 3 \mathrm{~km}$ depth beneath the Middle Mountain area. The change is shown most clearly in the $P$-to- $S$ scattering mode of the active source data and both the $P$-to- $S$ and $S$-to- $S$ scattering modes of the repeating earthquakes data, suggesting that the observed scattering property change is a result of charge or discharge of fluids in fractures caused by the 2004 Parkfield earthquake. The same scatterer(s) was found to respond to the 1993 aseismic stress/strain transient event, acting like an in situ stress-strain meter at seismogenic depth. Four-dimensional time-lapse imaging of the scattering wave field thus provides an effective way to monitor the subsurface stress-strain field.
\end{abstract}

\section{Introduction}

Monitoring the time-varying stress and strain field along an active fault has been a long-time goal of earthquake seismology. While the surface strain field is observable by geodetic instrumentation, such as Global Positioning System (GPS) and strainmeters, in situ measurements of the stress and strain field at seismogenic depth are still difficult to obtain. Laboratory studies show that elastic properties of crustal rocks can vary with applied stress as a result of opening or closing of fluid-filled cracks (Walsh, 1965; Scholz, 1968; Nur and Simmons, 1969; O’Connell and Budiansky, 1974). Thus, in principle, the stress field can be monitored by time-lapse (four-dimensional) seismic imaging of the elastic field, provided the scaling relationship between the two fields is well established. One approach to obtain such measurements is to examine temporal variations in the $P$-wave and $S$-wave coda wave field because they repeatedly sample the same region during their propagation and thus are more sensitive to subtle changes in the medium.

To perform time-lapse seismic imaging, a highly powerful and repeatable source, either natural or artificial, is required to minimize artifacts arising from changes of source location or source characteristics. One natural source is repeating earthquakes, which occur on the same patch of a fault plane with the same source mechanism at different times (e.g., Nadeau et al., 1995). When recorded at the same station, they produce highly similar seismograms. Repeating earthquakes have been widely used to identify coseismic velocity changes associated with large earthquakes (Schaff and Beroza, 2004; Rubinstein and Beroza, 2004a, 2004b, 2005; Peng and Ben-Zion, 2006; Rubinstein et al., 2007). Most of the observed coseismic velocity changes are found to occur at very shallow depth, usually less than a hundred meters, and are related to structural damage induced by strong ground motion. On the other hand, there are also reports of localized changes within the fault zone at seismogenic depth caused by stress transients associated with tectonic events (Niu et al., 2003; Taira et al., 2008).

Although repeating earthquakes are ideal sources to illuminate stress transients at seismogenic depth, given their depth and large size, it is difficult to use them to monitor 
these transients because of the lack of control on timing of the earthquakes and uncertainties in their absolute location. With the advantage of exactly known source characteristics and location, controlled sources such as explosions, air guns, and vibrators are more desirable in monitoring temporal changes of the velocity field, provided they generate sufficient energy to penetrate to seismogenic depths ( $\mathrm{Li}$ et al., 1998; Vidale and Li, 2003; Li et al., 2006). Studies of faultzone guide waves and $S$-coda waves by Li et al. $(1998,2003$, 2006) revealed a significant coseismic velocity drop inside a narrow 200-m fault damage zone and a logarithmic postseismic velocity healing. They further suggested that rock damages induced by dynamic rupture of the fault zone were responsible for the observed velocity change.

In this study, we use both natural and artificial sources to investigate temporal and spatial varying seismic scattering properties in the Parkfield region induced by the 2004 Parkfield earthquake (Langbein et al., 2005). An improved technique based on coda wave interferometry is developed to image sources of the temporal variations in coda waveform. We find a systematic pattern in temporal varying scattering properties (i.e., moving scatterers) at $\sim 3 \mathrm{~km}$ depth in the fault zone of the San Andreas fault from both the natural and artificial sources.

\section{Data}

The Parkfield section of the San Andreas fault is perhaps the best-instrumented region on the globe (Fig. 1). The region's High Resolution Seismic Network (HRSN), consisting of 13 borehole stations, provides an unprecedented opportunity to study the fine-scale seismic structure of the fault zone, as well as the evolving seismicity along the fault. The presence of repeating earthquakes (e.g., Nadeau et al., 1995) and the availability of about two decades of HRSN data make Parkfield an ideal place for studying temporal variations of fault properties. Figure 2 shows an example of the waveforms recorded at station VCA from a repeatingearthquake cluster, K03 (Nadeau and McEvilly, 1997; Nadeau and Johnson, 1998), which consists of 28 events occurring between 1988 and 2006. We analyzed HRSN recordings from four repeating-earthquake clusters to find coherent changes in the scattering field (Fig. 3 and Table 1). Note that the gray area in Figure 3 corresponds to an interrupted period of HRSN operation. No data were available during this period, which results in an apparent gap in repeating-earthquake detection. Niu et al. (2003) used earthquakes before the gap, and we mainly used the earthquakes after it. All four clusters include events that occurred a few months to one year before and immediately after the 2004 $M 6$ Parkfield earthquake, allowing us to investigate structural changes associated with that earthquake. Waveforms recorded at the HRSN stations from these four clusters are highly similar, suggesting that these clusters are tightly located. Double-difference relocations confirm this to within the relocations' uncertainties (i.e., $\sim 5-10 \mathrm{~m}$ ). In addition to

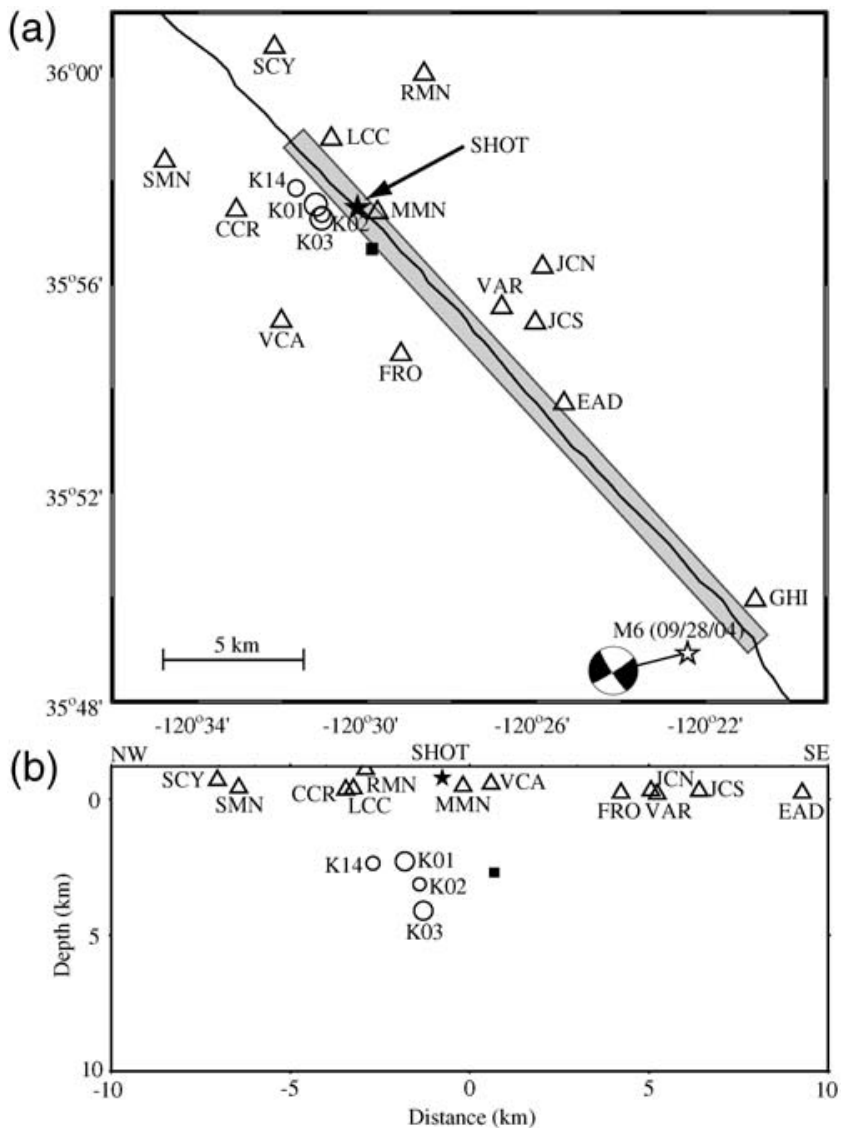

Figure 1. Map of the Parkfield study area: (a) map view, (b) fault cross-section view. The three-component-borehole High Resolution Seismic Network stations are shown as open triangles. The open circles show the locations of four repeating earthquake clusters used in this study. The size of each circle indicates the relative size of the earthquake. The black line shows the surface trace of the San Andreas fault. The black solid star shows the location of explosion shots. The black open star shows the hypocenter of the 2004 Parkfield $M 6$ earthquake. Also shown is the moment tensor solution and the surface rupture zone (gray area) for the 2004 Parkfield earthquake. The black solid square shows the location of the moving scatterer.

the passive repeating-earthquake data, we also used HRSN records of two 500-lb chemical explosions that were fired on 17 October 2002 and 29 December 2004. The two shots were separated by only $\sim 10 \mathrm{~m}$ and thereby generated very similar waveforms.

\section{Methods}

\section{Measuring Waveform Variations}

In this study, we analyzed only the vertical channel of the HRSN data, which usually has higher signal-to-noise ratio (SNR) than the two horizontal components. We first applied a second-order Butterworth band-pass filter (zero-phase shift) to the data. The pass band used was $5-35 \mathrm{~Hz}$ for repeatingearthquake data and $1-10 \mathrm{~Hz}$ for explosion data, respectively. We further interpolated the time sequences to 


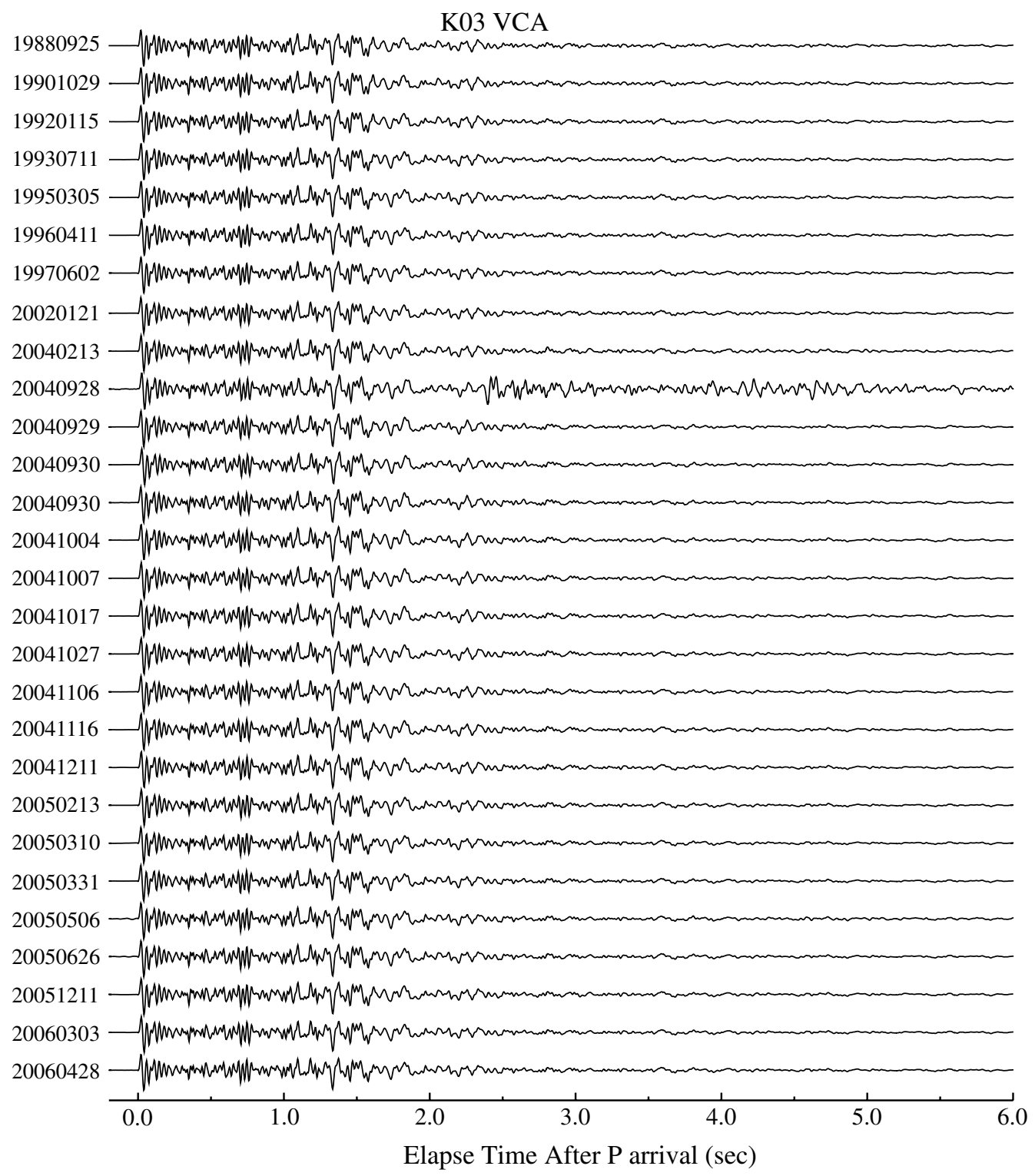

Figure 2. Example waveforms of repeating earthquakes. Shown are the vertical-component seismograms for repeating-earthquake cluster K03 at station VCA. Waveforms were aligned to the $P$ arrival time. Note the extreme waveform similarity throughout the entire 6-s time window, with the exception of the additional superposed aftershock occurring $\sim 2.5 \mathrm{~s}$ after the $P$ arrival on 28 September 2004 (20040928), 16 minutes after the 2004 Parkfield earthquake.

32, 000 samples/second to obtain better resolution in picking the arrival times. We estimated errors in delay time measurement based on the SNR of the data (Cheng et al., 2007) and used them to determine the sampling interval of the interpolation. Interpolation was performed in the frequency domain by padding zeros to the original spectra. We then computed the cross correlation between the first seismogram of each of the repeating-earthquake series and explosion pair with each subsequent seismogram in the corresponding sequence or pair to obtain the delay times of the $P$ arrivals between events of the sequences and explosions. We further used a cosine fitting method to estimate the subsample delay times that were not included from the cross-correlation method (De Jong et al., 1990; Cespedes et al., 1995). Given the largest sample of the correlation function $C(0)$ and its two neighbors $C(-1)$ and $C(1)$, the estimated subsample delay time is given by the following expression:

$$
\delta t=-\frac{\theta}{\omega_{0}} \cdot \Delta t
$$

where $\Delta t$ is the sampling interval, $\omega_{0}$ is the angular frequency of the cosine function given by

$$
\omega_{0}=\cos ^{-1}\left[\frac{C(-1)+C(1)}{2 C(0)}\right]
$$

and $\theta$ is the phase of the cosine function given by 


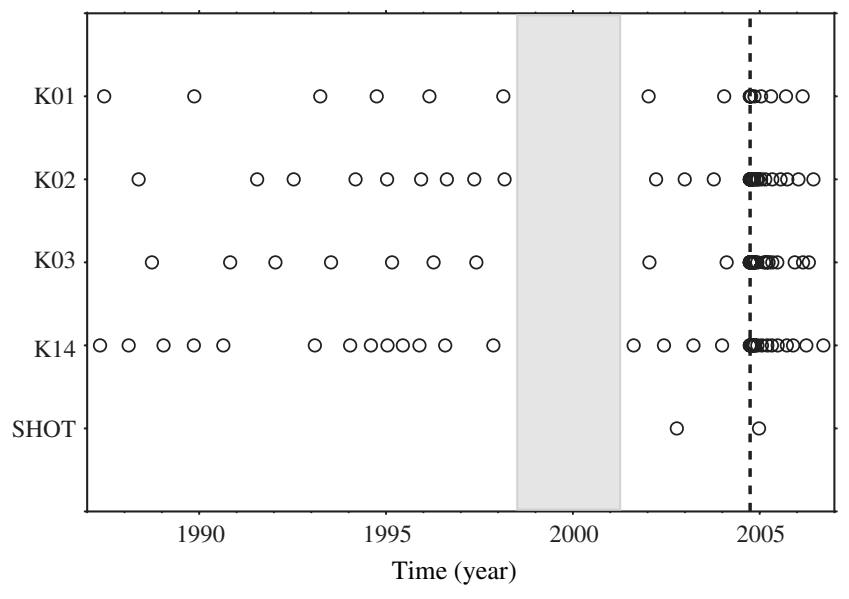

Figure 3. Time line of the four repeating-earthquake clusters (K01, K02, K03, K14) and two explosion shots used in this study. The vertical dashed line indicates the time of the Parkfield earthquake. The gray area corresponds to the period when HRSN stations went offline, which leaves a gap in the catalog of repeatingearthquake clusters.

$$
\theta=\tan ^{-1}\left[\frac{C(-1)-C(1)}{2 C(0) \sin \alpha}\right] .
$$

To ensure that all the seismograms were aligned at the peak cross correlation, we resampled the subsequent seismograms in each cluster with an eighth-order sinc function

$$
u^{\prime}(n \Delta t+\delta t)=\sum_{k=-8}^{8} u[(n+k) \Delta t] \frac{\sin [(\pi / \Delta t)(\delta t-k \Delta t)]}{(\pi / \Delta t)(\delta t-k \Delta t)},
$$

where $u(t)$ and $u^{\prime}(t)$ are the original and resampled time sequences, respectively. All the seismograms in each cluster were further normalized with their maximum amplitudes and aligned at the $P$-wave arrivals.

Once the seismograms were aligned, we computed the running time cross correlation between the first seismogram and each subsequent seismogram within a 0.5 -s moving time window:

$$
C(\tau(t), t)=\frac{\int_{t-T / 2}^{t+T / 2} u_{r}\left(t^{\prime}\right) u_{s}\left(t^{\prime}+\tau\right) d t^{\prime}}{\left[\int_{t-T / 2}^{t+T / 2} u_{r}^{2}\left(t^{\prime}\right) d t^{\prime} \int_{t-T / 2}^{t+T / 2} u_{s}^{2}\left(t^{\prime}\right) d t^{\prime}\right]^{1 / 2}},
$$

Table 1

Average Hypocenters and Magnitudes for Clusters Used*

\begin{tabular}{ccccc}
\hline Cluster & Latitude $\left({ }^{\circ}\right)$ & Longitude $\left(^{\circ}\right)$ & Depth $(\mathrm{km})$ & Magnitude \\
\hline K01 & 35.958961 & -120.520353 & 2.283 & 0.982 \\
K02 & 35.955837 & -120.517919 & 3.134 & 0.671 \\
K03 & 35.954549 & -120.517996 & 4.105 & 0.959 \\
K14 & 35.964190 & -120.527992 & 2.356 & 0.702 \\
Shot & 35.957916 & -120.503967 & -0.779 & \\
\hline
\end{tabular}

*Average hypocenters and magnitudes are estimated from event members occurring after 2001. Depth is in reference to mean sea level. where $u_{r}(t)$ and $u_{s}(t)$ are the first (reference) and the subsequent seismograms, respectively. The time window is centered at lapse time $t$ with a window length $T=0.5 \mathrm{~s}$. The $\operatorname{lag} \tau(t)$ was obtained when the maximum cross correlation $C_{\max }(\tau(t), t)$ was reached. We then defined a decorrelation index (Niu et al., 2003)

$$
D(t)=1-C_{\max }(\tau(t), t) .
$$

Here, $D(t)$ quantifies the dissimilarity between two seismograms and varies from 0 to 1 . If the subsequent seismogram is similar to the reference seismogram, then $D(t)=0$. For a medium with randomly distributed scatterers, the lag time $\tau(t)$ and the decorrelation index $D(t)$ are related to the weighted mean and variance of the time lags, $\tau_{i}$, associated with the individual scatterers in the time window centered on $t$ (Snieder et al., 2002):

$$
\tau(t)=\left\langle\tau_{i}\right\rangle ; \quad D(t)=\frac{1}{2} \omega^{2} \sigma_{\tau}^{2}(t)=\frac{1}{2} \omega^{2}\left(\left\langle\tau_{i}^{2}\right\rangle-\tau^{2}\right),
$$

where $\omega$ is the characteristic angular frequency of the scattered waves. According to equation (7), a change in the background velocity will result in a constant time $\operatorname{lag} \tau_{i}$ for all the scatterers, which increases linearly with the lapse time $t$ :

$$
\tau(t)=-\frac{\delta v}{v} \cdot t
$$

The constant time lags $\tau_{i}$ lead to a zero variance and subsequently a zero decorrelation index. Equation (8) has been widely used to infer coseismic velocity changes (Schaff and Beroza, 2004; Rubinstein and Beroza, 2005; Li et al., 2006; Rubinstein et al., 2007). $D(t)$ calculated from a perturbation in source location results in an initial increase with lapse time and quickly falls to a constant level (Snieder et al., 2002, Niu et al., 2003) that can be used as an indicator of the distance between the two sources. Snieder et al. (2005) used the constant level of the decorrelation index to investigate distances between earthquake doublets identified along the Hayward fault, California. On the other hand, a localized change in the scattering field should result in isolated spikes in both the lag time $\tau(t)$ and the decorrelation index $D(t)$ (Niu et al., 2003).

\section{Synthetic Tests}

Using numerical experiments, Niu et al. (2003) investigated the characteristics of $\tau(t)$ and $D(t)$ with changes in the source location, as well as in the bulk and localized velocity field. In their study, acoustic synthetics were computed and used in the numerical experiment. Here, we conducted the same experiments with two-dimensional (2D) elastic synthetics. We designed a 2D model with a dimension of 40 $\times 21.5 \mathrm{~km}$ and a set of 20-m spaced grids (Fig. 4). We used the perfectly matched layer absorbing boundary method 
(Berenger, 1996) for the four boundaries to reduce artificial reflections. A Ricker wavelet with a dominant frequency of $8 \mathrm{~Hz}$ was used as the source-time function. Following (Frankel and Clayton, 1986), we created a Gaussian random medium using

$$
R(r)=\varepsilon^{2} \exp \left(-r^{2} / a^{2}\right)
$$

where $R(r)$ is the autocorrelation function at distance $r, \varepsilon$ is the strength of the heterogeneity, and $a$ is the correlation distance or the scale of the heterogeneity. A Gaussian
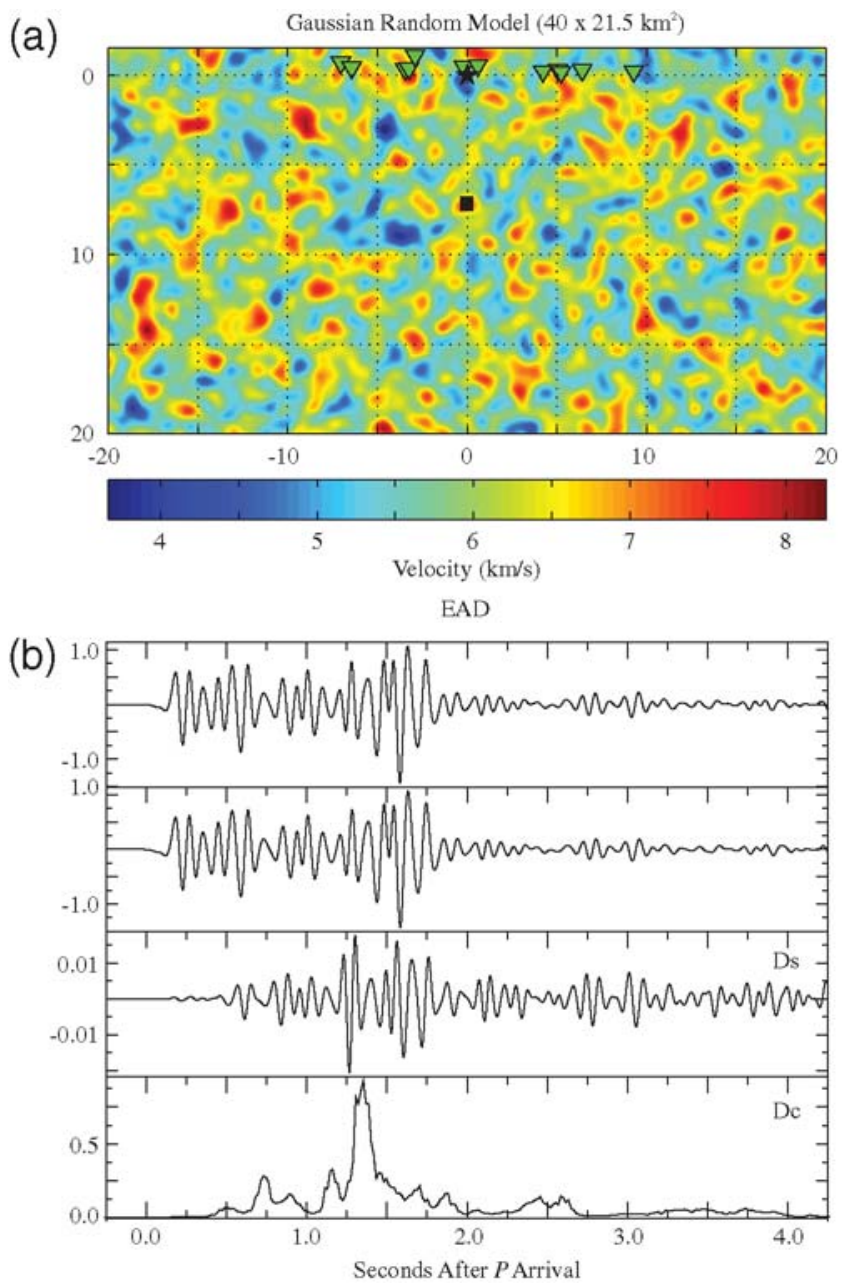

Figure 4. Finite difference forward modeling synthetic test. (a) A Gaussian random model was used to calculate the synthetic waveforms. A large model dimension helped ensure that no reflected artifacts were generated from the boundaries. The star shows the location of the simulated shot source. Triangles are the receivers mimicking the actual locations of the HRSN sensors. (b) The top two traces show examples of synthetic waveforms at station EAD before and after simultaneously introducing source location, background velocity, and localized velocity changes. The third and fourth traces are the resulting differential seismogram and running window decorrelation index measurements from the top two waveforms. Notice the decorrelation index measurements are normalized by the waveforms' SNR and that the scale of the differential seismograms is around one-tenth of the original waveforms. random medium with $6 \mathrm{~km} / \mathrm{s}$ mean, $0.6 \mathrm{~km} / \mathrm{s}$ standard deviation, and $1000 \mathrm{~m}$ correlation length was created as the velocity model. Because we used a 2D model, a velocity model with unrealistically high (10\%) perturbations was needed to generate the same level of coda energy shown in data originating from 3D scattering.

While Niu et al. (2003) found significant differences in $\tau(t)$ and $D(t)$ due to changes in source and medium, the purpose of our numerical experiment here is to determine whether or not we can identify localized changes in the medium when a change in the background velocity is also present and the two sources are loosely colocated. To do this, we have computed two sets of elastic synthetic seismograms with a source-receiver geometry that mimics the setting of the active source dataset shown in the Explosive Source Data section. The background velocity in the second model was $0.1 \%$ lower than in the first model, and the source was placed one grid $(20 \mathrm{~m})$ away from the source in the first model. We also introduced a strong scatterer with a dimension of $600 \times$ $600 \mathrm{~m}$ and a velocity contrast of $25 \%$ to the second model (Fig. 4a). The synthetic seismograms at station EAD calculated from the two models are shown in Figure 4. Although the two seismograms appeared to be very similar, substantial differences actually exist. This can be shown by constructing an apparent differential seismogram between the two. More importantly, the differences are mainly caused by the changes in the background velocity and source location, and it is almost impossible to identify the scattered coda waves associated with the strong scatterer from the apparent differential seismogram. On the other hand, the localized change in the scattering field can be easily recognized form the decorrelation index. Obviously, the $0.1 \%$ drop in the background velocity has relatively little effect on the decorrelation index.

\section{Locating Moving Scatterers with}

Diffraction Migration

To locate the scatterers for which the physical properties have changed in time, we constructed differential seismograms between the first and each subsequent event for each sequence and explosion pair by taking the difference of seismograms after taking account of the time lag:

$$
\delta u(t)=u_{s}[t+\tau(t)]-u_{r}(t) .
$$

In principle, only energy from time-dependent physical properties, such as changes in scatterer location, strength, or localized velocity change near to the scatterer, is expected to dominate in the differential seismograms. Energy from the unchanged background medium will be removed by this procedure. Note we have improved the method used by Niu et al. (2003) in computing the differential seismograms. The running time window-based subtraction is made between the two time windows with maximum cross correlation. Like the decorrelation index, this procedure can effectively 
remove the stretching effect that results from changes in background velocity.

We further applied a diffraction migration technique to the differential seismograms to locate the moving scatterers. To do this, we assumed a single scattering model and ignored the multiple scattering effect (Sato and Fehler, 1998). Using 3D $P$ - and $S$-wave velocity models derived from arrival times inversion for a combination of microearthquakes and surface Vibroseis sources (Michelini and McEvilly, 1991), we generated sets of $P$ - and $S$-wave travel time tables using a 3D eikonal solver (Zelt and Barton, 1998). The migrated image of potential moving scatterers was computed by

$$
I(\mathbf{r})=\sum_{s, i, j} \alpha_{s}^{m n} \delta u\left(t_{i j}^{m n}-t_{i j}^{P}\right)=\sum_{s, i, j} \alpha_{s}^{m n} \delta u\left(t_{i r}^{m}+t_{r j}^{n}-t_{i j}^{P}\right),
$$

where $m$ and $n$ represent the $P$ - and $S$-wave scattered modes with a combination of $P$-to- $P, P$-to- $S, S$-to- $P$, and $S$-to- $S$ converted phases; $t_{i j}^{m n}$ represents the total travel time of

(a)

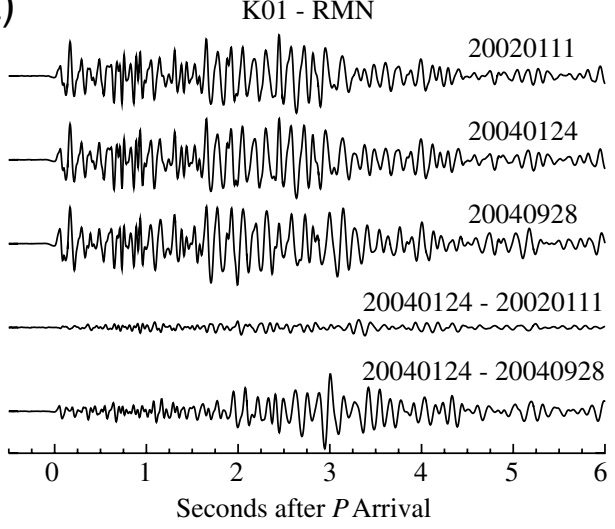

(c)

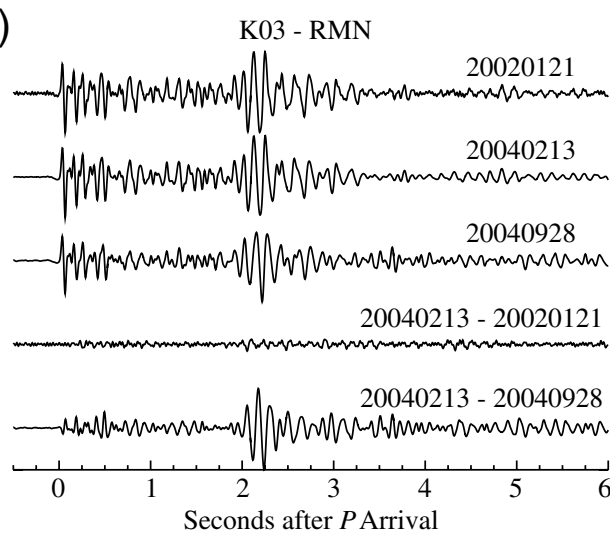

the $m n$ scattering wave, which was computed by summing the travel times from the $i$-th event to the scatterer located at $\mathbf{r}$ and from the scatterer to the $j$-th receiver; $t_{i j}^{P}$ is the arrival time of the direct $P$ wave; $a_{s}^{m n}$ is the weight function for each corresponding scattering mode. The summation was performed over all the event-station pairs, as well as all possible scattering modes.

\section{Results and Discussion}

\section{Repeating-Earthquake Data}

We have collected HRSN waveform data for four repeating-earthquake sequences to investigate changes in the scattering wave field associated with the 2004 M 6 Parkfield earthquake. These four repeating earthquakes clusters were used by Niu et al. (2003) to study the 1993 aseismic slip event in the same region. We first updated the catalog of the four clusters to the end of 2007.

Within each cluster, we selected the event immediately preceding the 2004 Parkfield earthquake as the reference

(b)

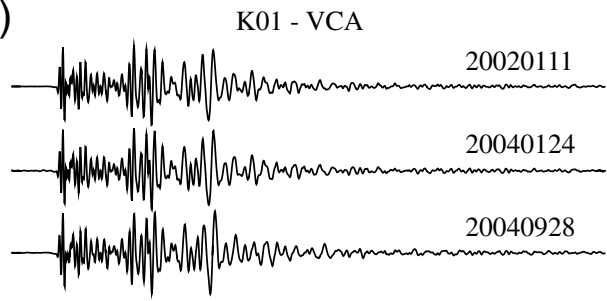

$20040124-20020111$

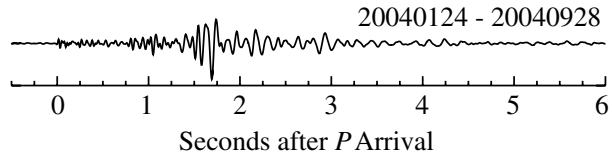

(d)

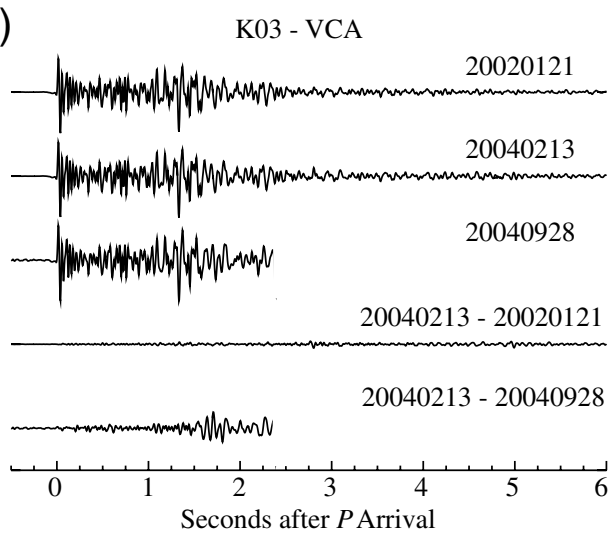

Figure 5. Example vertical-component waveforms of repeating-earthquake clusters and their corresponding differential seismograms. (a-d) Records of stations RMN and VCA for clusters K01 and K03. In each subplot, the top three traces show the waveforms before and immediate after the 2004 Parkfield earthquake. Dates are given in year, month, day (UTC). The bottom two traces show the differential seismograms constructed from waveforms before and across the occurrence time of the 2004 Parkfield earthquake. Clear energy emerges in the differential seismograms that span the occurrence time of the 2004 Parkfield earthquake, indicating the changes in coseismic scattering properties. In comparison, differential seismograms for the earlier two-year period that does not span the mainshock are very stable. Note that the seismogram from the VCA station for the K03 cluster event on 20040908 is cut after $2.5 \mathrm{~s}$ due to contamination by a superposed aftershock signal (see Fig. 2). 
(a)

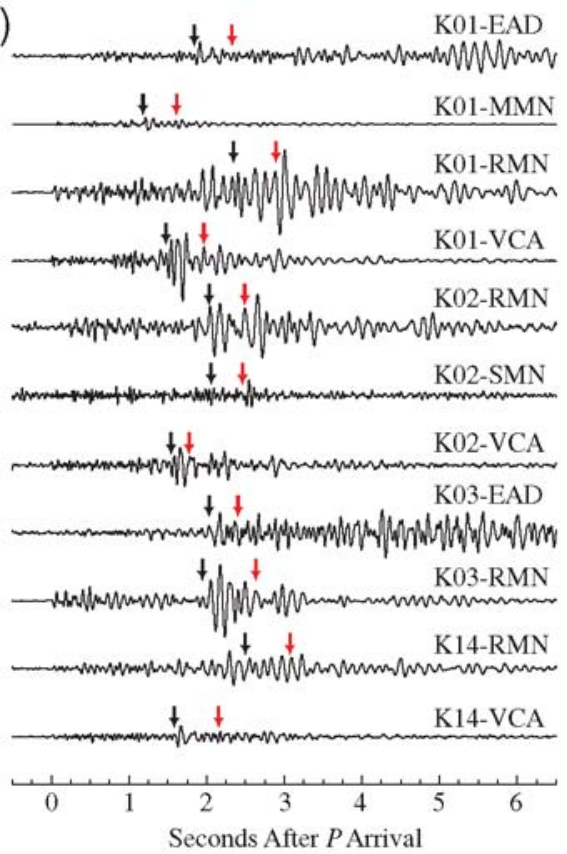

(b)

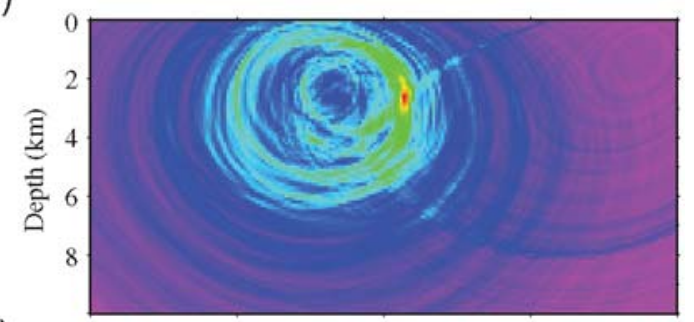

(c)

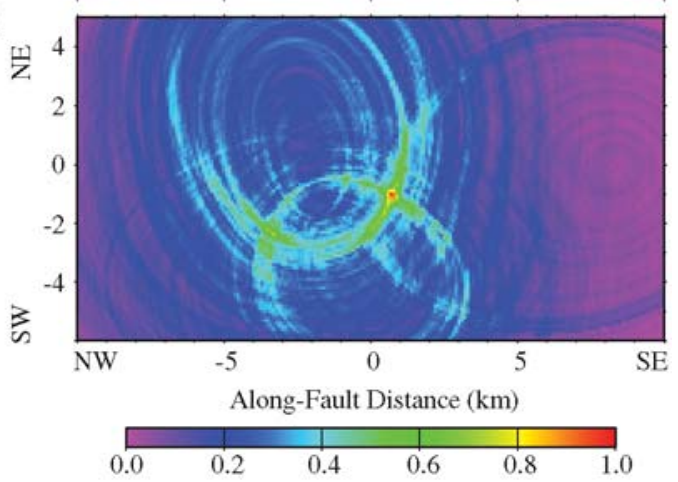

Figure 6. Differential seismograms and the imaging of scatterer migration. (a) Vertical-component differential seismograms, which were taken between the repeating events that most closely span the Parkfield earthquake for four repeating-earthquake clusters at several HRSN stations. (b) Cross-sectional view parallel to the fault and (c) a map view at 3-km depth. Using the differential seismograms shown in (a), the Born approximation migration results show a well-defined maximum amplitude area that indicates the location of the scattering region where the seismic scattering properties associated with the 2004 Parkfield earthquake showed a change. The color bar represents the normalized stacking differential seismogram amplitude used. The black and red arrows in (a) indicate the predicted arrival time for $P$-to- $S$ and $S$-to- $S$ converted phase from the moving scatterer.

event to calculate the time lag and the decorrelation index. Once the time lag for each time window was obtained, the differential seismograms were computed using equation (10). The reference events of the four clusters occurred a few months before the $M 6$ earthquake (Fig. 3); thus we have implicitly assumed that there were no significant changes in the scattering field during the several-month period before the $M 6$ earthquake. We argue that this is a reasonable assumption because very little energy was found in the differential seismograms constructed among events before the (a)

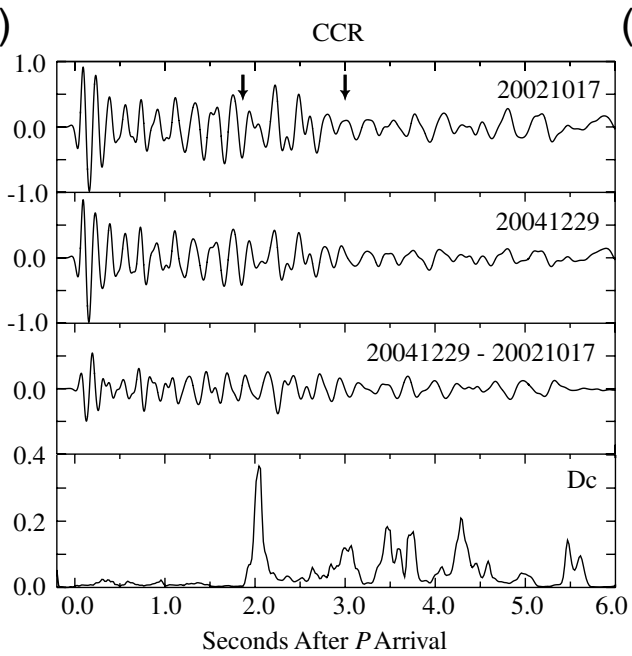

(b)

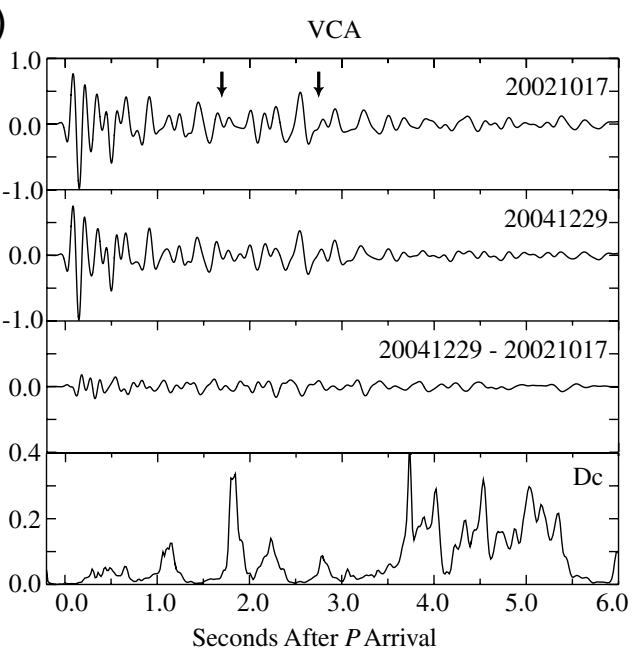

Figure 7. Example vertical-component waveforms of the two explosion shots. (a) and (b) show the shot recordings from stations CCR and VCA, respectively. Also shown are their corresponding differential seismograms and decorrelation index measurements. The arrows indicate the predicted arrival time for $P$-to- $S$ and $S$-to- $S$ converted phases from the moving scatterer observed in Figure 5. 
$M 6$ earthquake (Fig. 5). Figure 5 shows the differential seismograms recorded at stations RMN and VCA from clusters K01 and K03. For nearly two years prior to the Parkfield earthquake (between January of 2002 and January of 2004), no obvious differential energy was registered at either station from the K01 cluster. The same observation was also obtained for the differential seismograms constructed for events of cluster K03 with a two-year interval before the 2004 Parkfield earthquake. Meanwhile, we observed large and consistent changes in waveform between the event occurring in January 2004 and the one that was recorded 16 minutes after the $M 6$ earthquake. These changes were identified across all the four repeating-earthquake clusters and at all HRSN stations.

Rubinstein and Beroza (2005) found that the most significant coseismic velocity drop was located near the rupture area of the Parkfield earthquake and most of the velocity decreases occurred within the fault zone at very shallow depths $(<100 \mathrm{~m})$, due to direct damage caused by strong shaking during the earthquake. These shallow surface changes, however, appeared to have little affect on the borehole HRSN station recordings. In addition, the shallow changes appeared as changes in the overall background velocity, which our procedure is designed to remove from the differential seismograms and the decorrelation index.

We applied the diffraction migration technique described in the previous section (Locating Moving Scatterers with Diffraction Migration) to the differential seismograms calculated from the four clusters. Here the differential seismograms were computed from the two events occurring immediately before and after the $M 6$ earthquake. Because little energy was seen in the $P$-coda, we only used the $P$-to- $S$ and $S$-to- $S$ scattering modes to form the final image (Fig. 6). The $S$-wave coda energy in the differential seismograms was projected to a spot within the fault zone approximately $3 \mathrm{~km}$ below the Middle Mountain area (Fig. 6). The predicted time of the $P$-to- $S$ and $S$-to- $S$ scattered phases are marked with black and red arrows in Figure 6a. We interpret this spot as a transient scatterer(s) that responded to the $M 6$ earthquake. This scatterer also seems to be colocated with the scatterer identified by Niu et al. (2003). Therefore, it seems that this scatterer responded to both the 1993 aseismic event at Parkfield and the $2004 M 6$ Parkfield earthquake. Taira et al. (2009) also found that the same scatterer responded to the dynamic stress change of the $2004 M 9.1$ SumatraAndaman earthquake. Hence, this scatterer may be considered as a type of in situ stress change meter, providing the scaling relationship between the scattered wave field changes and the stress changes is well established.

\section{Explosive Source Data}

Two active-source experiments with chemical explosive sources were conducted in our study area on 17 October 2002 and 29 December 2004, crossing the occurrence time of the $2004 M 6$ Parkfield earthquake. The two explosions are almost colocated and thus produced highly similar seismograms at HRSN stations. Using fault-zone trapped waves generated by these two shots, Li et al. (2006) reported an $\sim 1 \%$ coseismic velocity drop within a $\sim 200$-m-wide fault zone along the fault strike. In general, records of surface instruments tend to be noisier, and it is very challenging to correct variations from the near-surface environment layer when they are used for time-lapse seismic imaging. We thus decided to use the HRSN records of the two explosions.
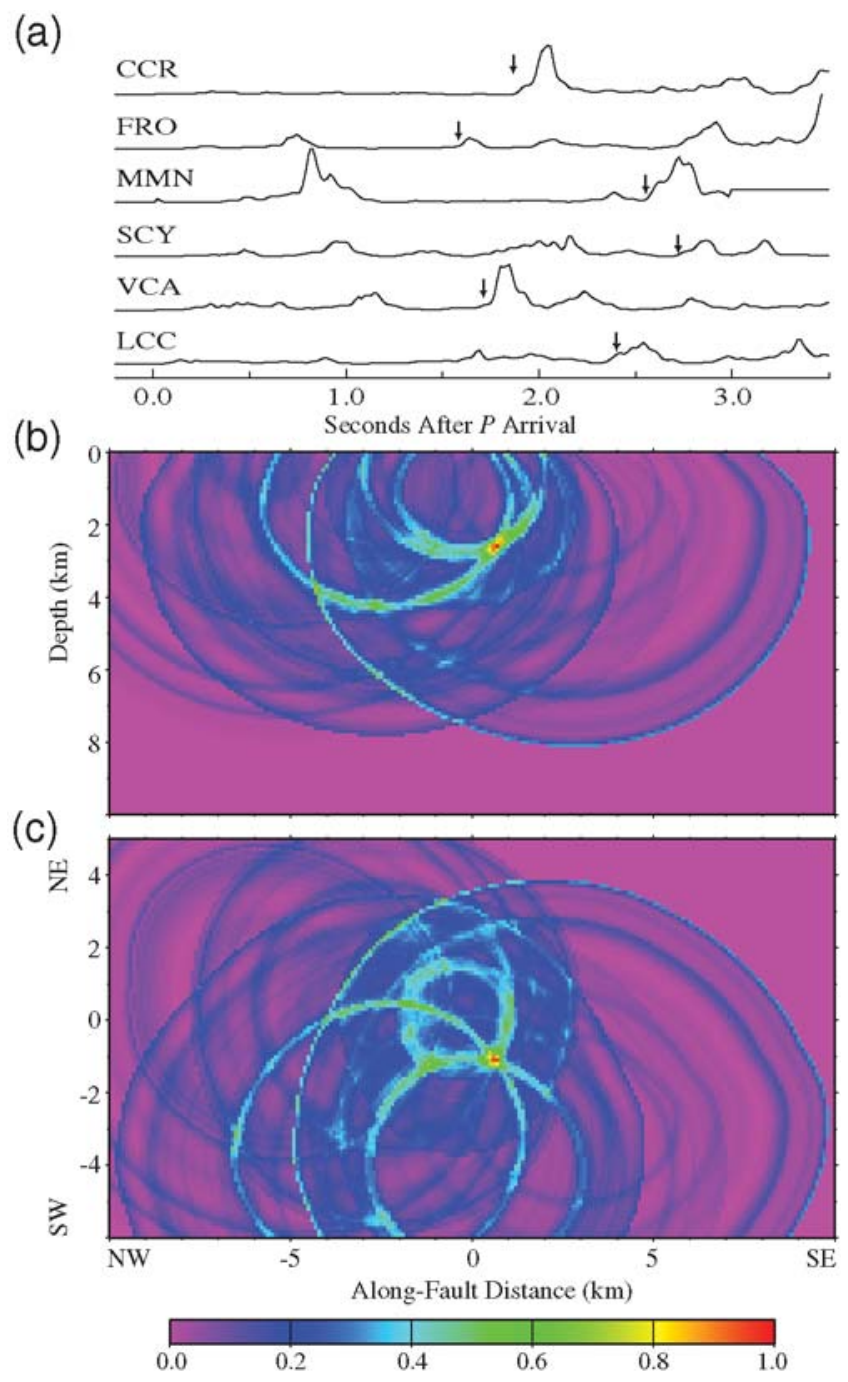

Figure 8. Running window decorrelation index measurements of the two explosion shots and the corresponding image of scatterer migration. (a) Running window decorrelation index measurements were calculated from vertical-component seismograms, which were taken between the shots before and after the Parkfield earthquake. (b) Cross-section view parallel to the fault and (c) a horizontal slice taken at $3 \mathrm{~km}$ depth. Using the decorrelation index measurements shown in (a), the Born approximation migration results show a consistent pattern of change in seismic scattering properties occurring at almost the same location as that found using the repeatingearthquake data. Color bar corresponds to normalized stacking decorrelation index. The arrows in (a) show the predicted arrival time for $P$-to- $S$ converted phase from the observed moving scatterer. 
We basically applied the same techniques and procedures used in analyzing the repeating-earthquake data. Seismograms of the first explosion were used as the reference for computation of the second event's lag time, the decorrelation index, and the differential seismograms.

Figure 7 shows examples of the differential seismograms and decorrelation indexes computed at stations CCR and VCA. Although the seismograms from the two shots are quite similar (Fig. 7), the similarity here is actually much lower than for the repeating-earthquake data. We attributed the lower similarity seen here to changes in the shot location, temporal changes in near-surface conditions at the shot site (e.g., water table, rainfall, etc.), and, more likely, to the large coseismic velocity drop within the nearsurface layer (Rubinstein and Beroza, 2005; Li et al., 2006) due to the Parkfield mainshock. Because the two shots were located near the fault zone at the surface, the downward wave-field propagation from the sources to the receivers may still sample the medium damage inside the shallow fault zone, despite borehole stations being used. We thus turned to the decorrelation index to identify potential transient scatterers. We also computed the arrival times of the $P$-to- $S$ and $S$-to- $S$ waves scattered at the transient scatterer (as identified by repeating earthquakes), and these arrivals are shown in Figure 7 with black arrows. We found distinct spikes in the decorrelation index within the predicted $P$-to- $S$ arrival time window. We expected the $S$-to- $S$ scattered waves to be relatively weak as very little $S$-wave energy radiated from the shots. We next back-projected the spikes in the decorrelation index, measured from all stations based on the $P$-to- $S$ scattering mode, into a 3D grid space. We found a well-defined location where the isochrones intersect with the others
(Fig. 8). The location matches well with the transient scatterer determined by the repeating-earthquake data. The predicted arrival times of the $P$-to- $S$ scattered wave are marked with black arrows on the running window decorrelation index time series in Figure 8a.

Baisch and Bokelmann (2001) reported coseismic changes in the scattering field associated with the 1989 M 6.9 Loma Prieta earthquake. They attributed the observed changes to coseismic deformation, which leads to crack opening either by local concentration of shear stress or by elevated pore pressure. Our results clearly show that the transient scatterer observed here is located at the edge of the area where significant coseismic slip from the Parkfield earthquake occurred (Johanson et al., 2006) (Fig. 9). Thus, the detailed structural change we see here is likely due to localized fluid redistribution on the San Andreas fault resulting from coseismic stress changes and fault-zone damage from the Parkfield earthquake.

\section{Conclusion}

We investigated temporal variations in the coda waveforms of four repeating-earthquake clusters and two repeating explosions and found a systematic change associated with the $2004 M 6$ Parkfield earthquake. Analysis of the decorrelation index revealed that a localized change in the scattering field was responsible for the observation. We improved the migration technique used in our previous study and applied it to both the earthquake and controlled source data. The two datasets revealed a consistent image of a timedependent scatterer, which is located $\sim 3 \mathrm{~km}$ deep beneath the Middle Mountain area at approximately the upper

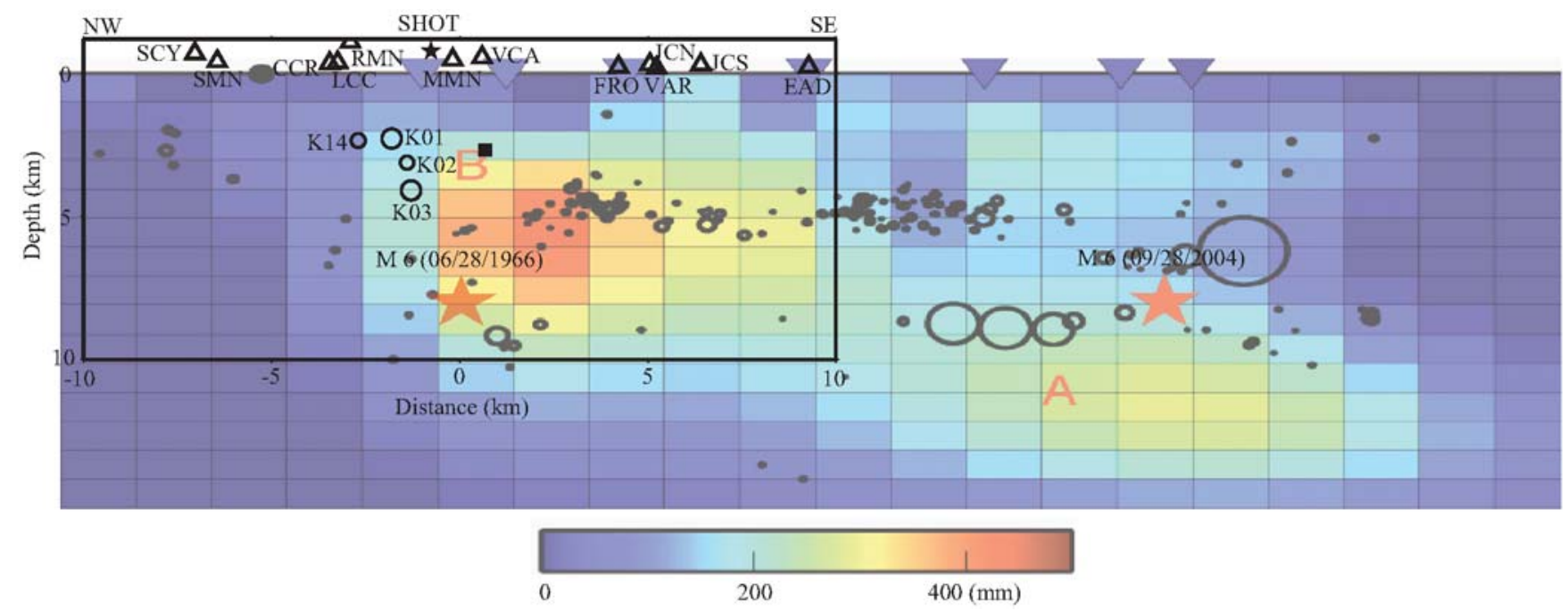

Figure 9. Comparison of this study's migration results from seismic data (upper figure; see Figure 1b for definitions of symbols) with a modified coseismic slip map from Johanson et al. (2006), which was inverted from GPS and InSAR data. Two red stars are the hypocenter of the 1966 and 2004 M 6 Parkfield earthquakes. The location of our observed moving scatterer is located at the edge of the area with strong coseismic slip, suggesting increased local stress caused by coseismic slip as the likely explanation for our observed scattering waveform variations. Background circles are the aftershocks of the 2004 Parkfield earthquake. The circle size is scaled to the size of the earthquakes. 
northwest edge of the major rupture zone of the $2004 M 6$ Parkfield earthquake. Assuming the wavelength of the scattered wave is comparable to the size of the scatterer, we estimate the dimension of the scatterer to be of the order of $300 \mathrm{~m}$, based on the characteristic frequency of the scattered wave $(10 \mathrm{~Hz})$ and local $S$-wave velocity $(3 \mathrm{~km} / \mathrm{s})$. The scatterer appears to be more efficient in scattering $S$-wave energy, indicating an involvement of fluid with the observed structural changes. Based on these observations, we speculated that the observed structural changes are likely caused by fluid redistribution resulting from a combination of stress relaxation and direct fault-zone damage. This is the third reported episode of stress change to which this scatterer has responded when examined using similar methods, confirming the scatterer as a robust indicator of stress change located directly in the seismogenic crust.

\section{Data and Resources}

Waveform data for this study were provided by the Northern California Earthquake Data Center (NCEDC).

\section{Acknowledgments}

Comments made by two anonymous reviewers were very helpful and significantly improved the manuscript. The Parkfield High-Resolution Seismic Network is operated by the University of California, Berkeley Seismological Laboratory, with financial support from the US Geological Survey (USGS) through awards 07HQAG0014 and G10AC00093.

\section{References}

Baisch, S., and G. H. R. Bokelmann (2001). Seismic waveform attributes before and after the Loma Prieta earthquake: Scattering change near the earthquake and temporal recovery, J. Geophys. Res. 106, $16,323-16,337$.

Berenger, J. P. (1996). Three-dimensional perfectly matched layer for the absorption of electromagnetic waves, J. Comput. Phys. 127, 363-379.

Cespedes, I., Y. Huang, J. Ophir, and S. Spratt (1995). Methods for estimation of sub-sample time delays of digitized echo signals, Ultrason. Imaging 17, 142-171.

Cheng, X., F. Niu, P. G. Silver, S. Horiuchi, K. Takai, Y. Iio, and H. Ito (2007). Similar microearthquakes observed in western Nagano, Japan, and implications for rupture mechanics, J. Geophys. Res. 112, no. B04306, doi 10.1029/2006JB004416.

De Jong, P. G. M., T. Arts, A. P. G. Hoeks, and R. S. Reneman (1990). Determination of tissue motion velocity by correlation interpolation of pulsed ultrasonic echo signals, Ultrason. Imaging 12, 84-98.

Frankel, A., and R. W. Clayton (1986). Finite-difference simulations of seismic scattering: Implications for propagation of short-period seismic waves in the crust and models of crustal heterogeneity, J. Geophys. Res. 91, 6465-6489.

Johanson, I. A., E. J. Fielding, F. Rolandone, and R. Burgmann (2006). Coseismic and postseismic slip of the 2004 Parkfield earthquake from space-geodetic data, Bull. Seismol. Soc. Am. 96, 269-282, doi 10.1785/0120050818.

Langbein, J., R. Borcherdt, D. Dreger, J. Fletcher, J. L. Hardebeck, M. Hellweg, C. Ji, M. Johnston, J. R. Murray, R. Nadeau, M. J. Rymer, and J. A. Treiman (2005). Preliminary report on the 28 September M 6.0 Parkfield, California earthquake, Seismol. Res. Lett. 76, 10-26.

Li, Y.-G., P. Chen, E. S. Cochran, J. E. Vidale, and T. Burdette (2006). Seismic evidence for rock damage and healing on the San Andreas fault associated with the 2004 M 6 Parkfield earthquake, Bull. Seismol. Soc. Am. 96, 349-363.

Li, Y.-G., J. E. Vidale, K. Aki, F. Xu, and T. Burdette (1998). Evidence of shallow fault zone healing after the 1992 M 7.5 Landers, California, earthquake, Science 279, 217-219.

Li, Y.-G., J. E. Vidale, S. M. Day, D. D. Oglesby, and E. Cochran (2003). Postseismic fault healing on the rupture zone of the 1999 M 7.1 Hector Mine, California, earthquake, Bull. Seismol. Soc. Am. 93, 854-869.

Michelini, A., and T. V. McEvilly (1991). Seismological studies at Parkfield, I: Simultaneous inversion for velocity structure and hypocenters using cubic B-splines parameterization, Bull. Seismol. Soc. Am. 81, 524-552.

Nadeau, R. M., and L. R. Johnson (1998). Seismological studies at Parkfield, VI: Moment release rates and estimates of source parameters for small repeating earthquakes, Bull. Seismol. Soc. Am. 88, 790-814.

Nadeau, R. M., and T. V. McEvilly (1997). Seismological studies at Parkfield, V: Characteristic microearthquake sequences as fault-zone drilling targets, Bull. Seismol. Soc. Am. 87, 1463-1472.

Nadeau, R. M., W. Foxall, and T. V. McEvilly (1995). Clustering and periodic recurrence of microearthquakes on the San Andreas fault at Parkfield, California, Science 267, 503-507.

Niu, F., P. G. Silver, R. M. Nadeau, and T. V. McEvilly (2003). Stressinduced migration of seismic scatterers associated with the 1993 Parkfield aseismic transient event, Nature 426, 544-548.

Nur, A., and G. Simmons (1969). The effect of saturation on velocity in low porosity rocks, Earth Planet. Sci. Lett. 7, 183-193.

O'Connell, R. J., and B. Budiansky (1974). Seismic velocities in dry and saturated cracked solids, J. Geophys. Res. 79, 5412-5426.

Peng, Z., and Y. Ben-Zion (2006). Temporal changes of shallow seismic velocity around the Karadere-Düzce branch of the north Anatolian fault and strong ground motion, Pure Appl. Geophys. 163, 567-599, doi 10.1007/s00024-005-0034-6.

Rubinstein, J. L., and G. C. Beroza (2004a). Evidence for widespread nonlinear strong ground motion in the $M_{\mathrm{w}} 6.9$ Loma Prieta earthquake, Bull. Seismol. Soc. Am. 94, 1595-1608.

Rubinstein, J. L., and G. C. Beroza (2004b). Nonlinear strong ground motion in the $M_{\mathrm{L}}$ 5. 4 Chittenden earthquake: Evidence that preexisting damage increases susceptibility to further damage, Geophys. Res. Lett. 31, L23614, doi 10.1029/2004GL021357.

Rubinstein, J. L., and G. C. Beroza (2005). Depth constraints on nonlinear strong ground motion from the 2004 Parkfield earthquake, Geophys. Res. Lett. 32, L14313, doi 10.1029/2005GL023189.

Rubinstein, J. L., N. Uchida, and G. C. Beroza (2007). Seismic velocity reductions caused by the 2003 Tokachi-Oki earthquake, J. Geophys. Res. 112, no. B05315, doi 10.1029/2006JB004440.

Sato, H., and M. C. Fehler (1998). Seismic Wave Propagation and Scattering in the Heterogeneous Earth, Springer, New York, 308 pp.

Schaff, D. P., and G. C. Beroza (2004). Coseismic and postseismic velocity changes measured by repeating earthquakes, J. Geophys. Res. 109, no. B10302, doi 10.1029/2004JB003011.

Scholz, C. H. (1968). Microfracturing and the inelastic deformation of rock in compression, J. Geophys. Res. 73, 1417-1432.

Snieder, R., and M. Vrijlandt (2005). Constraining the source separation with coda wave interferometry: Theory and application to earthquake doublets in the Hayward fault, California, J. Geophys. Res. 110, no. B04301, doi 10.1029/2004JB003317.

Snieder, R., A. Grêt, H. Douma, and J. Scales (2002). Coda wave interferometry for estimating nonlinear behavior in seismic velocity, Science 295, 2253-2255, doi 10.1126/science.1070015.

Taira, T., P. G. Silver, F. Niu, and R. M. Nadeau (2008). Detecting seismogenic stress evolution and constraining fault zone rheology in the San Andreas fault following the 2004 Parkfield earthquake, J. Geophys. Res. 113, no. B03303, doi 10.1029/2007JB005151.

Taira, T., P. G. Silver, F. Niu, and R. M. Nadeau (2009). Remote triggering of fault-strength changes on the San Andreas fault at Parkfield, Nature 461, 636-639, doi 10.1038/Nature08395. 
Vidale, J. E., and Y. G. Li (2003). Damage to the shallow Landers fault from the nearby Hector Mine earthquake, Nature 421, 524-526.

Walsh, J. B. (1965). The effect of cracks on the compressibility of rock, J. Geophys. Res. 70, 381-389.

Zelt, C. A., and P. J. Barton (1998). 3D seismic refraction tomography: A comparison of two methods applied to data from the Faeroe Basin, J. Geophys. Res. 103, 7187-7210.

Department of Earth Science

Rice University

6100 Main Street

Houston, TX 77005

xchengseis@gmail.com

(X.C., F.N.)
Department of Terrestrial Magnetism

Carnegie Institution of Washington

5241 Broad Branch Road, N.W.

Washington, D.C. 20015

(P.G.S.)

Berkeley Seismological Lab

University of California

207 McCone Hall

Berkeley, California 94720

(R.M.N.)

Manuscript received 25 September 2010 